\title{
Parallel Optimization Methods Based on Direct Search
}

\author{
Rafael A. Trujillo Rasúa ${ }^{1,2}$, Antonio M. Vidal ${ }^{1}$, \\ and Víctor M. García ${ }^{1}$ \\ ${ }^{1}$ Departamento de Sistemas Informáticos y Computación \\ Universidad Politécnica de Valencia \\ Camino de Vera s/n, 46022 Valencia, España \\ $\{$ rtrujillo, avidal, vmgarcia\}@dsic.upv.es \\ 2 Departamento de Técnicas de Programación, \\ Universidad de las Ciencias Informáticas, \\ Carretera a San Antonio de los Baños Km 2 s/n, \\ La Habana, Cuba \\ trujillo@uci.cu
}

\begin{abstract}
This paper is focused in the parallelization of Direct Search Optimization methods, which are part of the family of derivative-free methods. These methods are known to be quite slow, but are easily parallelizable, and have the advantage of achieving global convergence in some problems where standard Newton-like methods (based on derivatives) fail. These methods have been tested with the Inverse Additive Singular Value Problem, which is a difficult highly nonlinear problem. The results obtained have been compared with those obtained with derivative methods; the efficiency of the parallel versions has been studied.
\end{abstract}

\section{Introduction}

The general unrestricted optimization problem can be stated as follows: Given a continuous function $f: \Re^{n} \longrightarrow \Re$, find the minimum of this function. The most popular optimization methods use derivative or gradient information to build descent directions. However, there are many situations where the derivatives may not exist or cannot be computed, or are too expensive to compute. In such situations the derivative-free methods can be the only resource. These methods were studied first by Hooke and Jeeves [5] in 1961. In 1965 another important method of this kind was discovered, the Nelder-Mead simplex method [11.

All these methods are known to be fairly robust and locally convergent, but they are also quite slow, compared with derivative methods. For some time, this caused a lack of interest in these methods. However, with the advent of parallel computing these methods were again popular, since they are easily parallelizable [13, 2, 6].

This paper address the parallelization of a subset of these methods, termed in [9] as "Direct Search methods", where these methods are deeply described and studied. For the work described in this paper, different versions of these Direct 
Search methods have been implemented and parallelized; in this paper the best versions are presented, and, as expected, the parallel versions obtain substantial time reductions over the sequential versions. Still more remarkable is the overall robustness of the direct search methods. These methods have been tested with a very difficult problem, the Inverse Additive Singular Value Problem (IASVP), which is a problem where Newton-like methods usually fail to converge, even using globalization techniques as Armijo's rule 7]. As will be shown, the Direct Search methods converge where Newton's method fails, although they are still substantially slower than Newton's method.

The next section is devoted to the description of the Direct Search methods and the sequential versions implemented. Next, the parallel versions are presented, followed by the numerical results of the sequential and parallel methods. Finally the conclusions shall be given.

\section{Direct Search Methods}

We will present two different direct search methods; both belong to the class of Generating Set Search (GSS), as defined by Kolda et. al. in [9]. These methods start from an initial point $x_{0}$, an initial step length $\Delta_{0}$ and a set of directions spanning $\Re^{n}: D_{0}=\left\{d_{i}\right\}_{i=1, \ldots p}, d_{i} \in \Re^{n}$, so that every vector in $\Re^{n}$ can be written as a nonnegative linear combination of the directions in $D_{k}$.

The driving idea of the GSS methods is to find a decrease direction among those of $D_{k}$. To do that, in each iteration $k$ the objective function $f$ is evaluated along the directions in $D_{k}$. At that stage, the actual point shall be $x_{k}$ and the step length is $\Delta_{k}$; therefore, $f\left(x_{k}+\Delta_{k} d_{i}\right)$ is computed, for $i=1, \ldots, p$, until a direction $d_{i}$ is found such that $f\left(x_{k}+\Delta_{k} d_{i}\right)<f\left(x_{k}\right)$. If no such direction is found, the step length is decreased and the function is evaluated again along the directions.

When an acceptable pair $\left(\Delta_{k}, d_{i}\right)$ is found, the new point is updated:

$\left(x_{k+1}=x_{k}+\Delta_{k} d_{i}\right)$, a new step length $\Delta_{k+1}$ is computed and the set of directions is possibly modified or updated. This procedure shall be repeated until convergence (that is, when the step length is small enough).

This general algorithmic framework can be implemented in many ways.Among the versions that we have implemented, we have chosen the two versions described below, since they give the best results.

\subsection{Method GSS1}

In our first version, the direction set chosen as

$$
D_{k}=\left\{ \pm e_{i}\right\} \cup\{(1,1, \cdots, 1),(-1,-1, \cdots,-1)\},
$$

that is, the set of the coordinate axes and the vectors $(1,1, \cdots, 1)$ and $(-1,-1, \cdots,-1)$ which very often accelerate the search when the initial point is far from the optimum. 
Another characteristic is the strategy proposed in [6] of doubling the step length when the same descent direction is chosen in two consecutive iterations.

A distinct characteristic of our method (not proposed before, as far as we know) is the appropriate rotations of the set of directions. The evaluations are carried out in the order in which the directions are located in $D_{k}$. Therefore, if the descent directions are located in the first positions of the vector, the algorithm should find the optimum faster. To accomplish that, we propose that if in the iteration $k$ the first descent direction is $d_{i}$, it means that $d_{1}, \ldots, d_{i-1}$ are not descent directions and, most likely, will not be descent directions in the next iterations. Therefore, in our algorithm, these directions are displaced to the end of the $D_{k}$ set; that is, the new set would be: $\left(d_{i}, d_{i+1}, \ldots, d_{n}, d_{1}, \ldots, d_{i-1}\right)$.

\subsection{Method GSS2}

This method follows a similar strategy to the described by Hooke and Jeeves in 5. Here the initial direction set is $D_{0}=\left\{ \pm e_{i}\right\}$, and, unlike in GSS1, all the directions in $D_{k}$ are explored. Then, choosing all the descent directions: $d_{i, 1}^{(k)}, d_{i, 2}^{(k)}, \ldots d_{i, j}^{(k)}$, a new descent direction is built:

$$
d=d_{i, 1}^{(k)}+d_{i, 2}^{(k)}+\ldots+d_{i, j}^{(k)}
$$

which probably will cause a greater descent. If this is not true, the algorithm will choose among $d_{i, 1}^{(k)}, d_{i, 2}^{(k)}, \ldots d_{i, j}^{(k)}$ the direction with larger descent.

Clearly, this algorithm should need less iterations for convergence than GSS1, but the cost of each iteration is larger, since the function will be evaluated along all the directions.

\section{Parallelization of the Direct Search Methods}

The simplicity of the direct search methods and the independence of the function evaluations along each direction makes the parallelization of these methods a relatively easy task. The only serious problem to solve is the load imbalance that can suffer the algorithm GSS1. We will describe first the simpler parallel version of GSS2, and then two different options for GSS1.

\subsection{Parallel Method PGSS2}

The $2 n$ function evaluations needed for each iteration of GSS2 can be distributed among $p$ processors, so that each one carries out approximately $\frac{2 n}{p}$ evaluations. The parallel algorithm PGSS2 would start by distributing the initial point $x_{0}$, the initial step length $\Delta_{0}$ and a set of approximately $\frac{2 n}{p}$ directions. In each iteration each processor would evaluate the function along each direction, and would return the descent directions found and the corresponding function values to the "Root" processor. This processor would form the new direction (as in (2) ), would evaluate the function along this direction and obtain the new point $x_{k+1}$ and the new step length $\Delta_{k+1}$, which would be broadcasted to the other processors. 
In this algorithm PGSS2 there is hardly any load imbalance, and few communications, so that the speed-up is expected to be close to the optimum.

\subsection{Parallel Method PGSS1}

The parallel version of the algorithm GSS1 follows the same data distribution of GSS2. However, the underlying strategy must be different. Let us recall that in the GSS1 algorithm the function is evaluated sequentially along each direction until a descent direction is found.

As in the parallel version of GSS2, the directions are distributed among the processors. If a processor finds among its directions a descent one, it should warn the rest of processors to stop searching. The strategy chosen for this parallel algorithm is to select a number of evaluations $m$ that any processor should perform before reporting to the other processors. If after $m$ evaluations there has been no success, they must perform $m$ evaluations more, and repeat the process until a descent direction is found or they run out of directions.

It might happen that when the processors broadcast their results, several descent directions are found. If this happens, the direction of larger descent is chosen. This can cause that the parallel algorithm PGSS1 can make less iterations than the sequential version GSS1.

\subsection{Parallel Method Master-Slave MSPGSS1}

In the section 3.2 the algorithm described will perform a number of innecesary evaluations, depending on the value of $m$, and maybe some innecesary communications (if $m$ is too small). As an attempt to improve it, we implemented an asynchronous parallel version, using a Master-Slave scheme.

The Master processor controls all the search, while the slaves perform the evaluations of the function along the directions. The directions are distributed among the slaves; after each evaluation, the slave sends the value with a nonblocking message to the master processor. The non-blocking message allows that the slave goes on with its work. When the master detects a descent, sends to all the slaves a Success message; then, computes the new point and step length, and broadcasts it to all the slaves.

A drawback of this algorithm is that there is one processor less to perform evaluations, although this allows a better control and overlaps computing time with communication time.

\section{Experimental Results}

\subsection{Sequential Experiments}

All these algorithms have been implemented and applied to a difficult problem, the Inverse Additive Singular Value Problem (IASVP), which can be defined as: 
Given a set of matrices $A_{0}, A_{1}, \ldots, A_{n} \in \Re^{m \times n}(m \geq n)$ and a set of real numbers $S^{*}=\left\{S_{1}^{*}, S_{2}^{*}, \ldots, S_{n}^{*}\right\}$, where $S_{1}^{*}>S_{2}^{*}>\ldots>S_{n}^{*}$, find a vector $c=$ $\left[c_{1}, c_{2}, \ldots, c_{n}\right]^{t} \in \Re^{n}$, such that $S^{*}$ are the singular values of

$$
A(c)=A_{0}+c_{1} A_{1}+\ldots+c_{n} A_{n} .
$$

This problem is usually formulated as a nonlinear system of equations, and is solved with different formulations of Newton's method; several formulations are analyzed in 3. However, it can be formulated as well as an optimization problem, minimizing the distance between the desired singular values $S^{*}$ and the singular values of $A(c)$.

The sequential algorithms were implemented in C, using Blas [4] and LAPACK [1], and were executed in a $2 \mathrm{GHz}$ Pentium Xeon with 1 GByte of RAM and with operating system Red Hat Linux 8.0. For the experiments carried out with sequential algorithms, random square matrices were generated with sizes $n=$ $5,10,15,20,25,30,40,50$; the solution vector $c^{*}$ was chosen randomly as well, and the initial guesses $c_{i}^{(0)}$ were taken perturbing the solution with different values $\delta: c_{i}^{(0)}=c^{*}+\delta, i=1, \cdots, n$.

To obtain a fair appreciation of the performance of the Direct Search methods applied to the IASVP, the results of these methods were compared to Newton's method. Since there was convergence problems, Armijo's rule used to improve convergence in Newton's method. However, it became clear that Newton's method is very sensitive to the distance from the initial point to the solution. When the perturbation $\delta$ used was small, $\delta=0.1$ or smaller, Newton's method converged always. However, when $\delta=1.1$ it only converged in the case with $n=5$, and when $\delta=10.1$ it did not converge in any test. Meanwhile, the Direct Search methods converged in all the cases, although in a quite large number of iterations.

Table 1. Number of iterations to convergence, $\delta=0.1$

\begin{tabular}{|c|cccccccc|}
\hline$n$ & 5 & 10 & 15 & 20 & 25 & 30 & 40 & 50 \\
\hline GSSI & 82 & 630 & 2180 & 967 & 827 & 6721 & 7914 & 7178 \\
GSSII & 51 & 669 & 611 & 372 & 322 & 2237 & 1368 & 7292 \\
Newton & 3 & 3 & 7 & 5 & 7 & 7 & 5 & 6 \\
\hline
\end{tabular}

In the table 2 are shown the execution times of GSS1, GSS2 and Newton's method for the case $\delta=0.1$, when Newton's method converges. GSS1 is faster than GSS2, but Newton's method is much faster than Direct Search methods, when it converges.

Table 2. Execution Times (seconds), $\delta=0.1$

\begin{tabular}{|c|cccccccc|}
\hline$n$ & 5 & 10 & 15 & 20 & 25 & 30 & 40 & 50 \\
\hline GSSI & 0,03 & 1,26 & 3,37 & 2,80 & 4,10 & 45,00 & 139,76 & 237,48 \\
GSSII & 0,02 & 0,50 & 2,96 & 4,12 & 6,77 & 86,21 & 170,34 & 2054,88 \\
Newton & 0,00 & 0,00 & 0,01 & 0,01 & 0,02 & 0,04 & 0,08 & 0,25 \\
\hline
\end{tabular}


These results show that the application of the sequential Direct Search methods to high complexity problems is not practical, since the execution times are too high. However, they show a very interesting property; at least for this problem, they are far more robust than Newton's method when the initial guess is far away from the solution.

\subsection{Parallel Experiments}

The tests were carried out in a cluster with $202 \mathrm{GHz}$ Intel Xeon biprocessors, each one with 1 Gbyte of RAM, disposed in a 4x5 mesh with 2D torus topology and interconnected through a SCI network. The parallel methods were implemented using MPI [12. The sizes of the problems were conditioned by the high cost of the sequential solution. Due to this, the sizes of the problems were not too large: $n=72,96,120,144$. However, it must be noted that Newton's algorithm did not converge in these cases, even taking a initial guess very close to the real solution. The tables 345] summarize the execution times of the parallel algorithms.

Table 3. Execution Times (seconds) PGSS1

\begin{tabular}{|c|ccccccc|}
\hline size \processors & 1 & 2 & 4 & 6 & 8 & 10 & 16 \\
\hline 72 & 763 & 307 & 136 & 80 & 59 & 64 & 32 \\
96 & 3929 & 1982 & 621 & 362 & 336 & 266 & 187 \\
120 & 10430 & 4961 & 1891 & 943 & 669 & 605 & 364 \\
144 & 36755 & 17935 & 5626 & 3187 & 2209 & 1599 & 1207 \\
\hline
\end{tabular}

Table 4. Execution Times (seconds) PGSS2

\begin{tabular}{|c|ccccccc|}
\hline size $\backslash$ processors & 1 & 2 & 4 & 6 & 8 & 10 & 16 \\
\hline 72 & 278 & 141 & 72 & 50 & 38 & 33 & 23 \\
96 & 2710 & 1400 & 710 & 482 & 375 & 313 & 210 \\
120 & 5020 & 2581 & 1322 & 897 & 703 & 560 & 377 \\
144 & 14497 & 7349 & 3749 & 2537 & 1948 & 1565 & 1028 \\
\hline
\end{tabular}

Table 5. Execution Times (seconds) MSPGSS1

\begin{tabular}{|c|ccccccc|}
\hline size $\backslash$ processors & 1 & 2 & 4 & 6 & 8 & 10 & 16 \\
\hline 72 & 763 & 762 & 214 & 133 & 95 & 108 & 58 \\
96 & 3929 & 3940 & 1228 & 767 & 467 & 482 & 263 \\
120 & 10430 & 10614 & 3494 & 2108 & 1459 & 1134 & 958 \\
144 & 36755 & 36786 & 12258 & 6244 & 4375 & 3630 & 2286 \\
\hline
\end{tabular}

It must be observed that the sequential algorithm is the same for PGSS1 and MSPGSS1, and therefore the times are identical. These times are quite similar as well to the times of the MSPGSS1 with two processors, since there is only a slave processor performing the evaluations. 
The Master-slave algorithm performs worse than the other two. The fact of having one less processor performing evaluations does not seem to be the reason, since with 10 or 16 processors the influence should be less important.

The comparison of the algorithms PGSS1 and PGSS2 shows interesting trends; for these new sizes the sequential algorithm GSS1 deteriorates quite fast when the problem size grows, unlike in the smaller test cases (See Table 2). With only one processor, the algorithm GSS2 is twice faster for large problems.

However, when the number of processors increases the situation changes; the PGSS2 algorithm gives good speedups, as expected, but the PGSS1 algorithm obtains great advantages when the number of processors grows, so that for large number of processors it obtains execution times close to those of PGSS2. It can be seen that the algorithm PGSS1 obtains speed-ups over the theoretical maximum, which is the number of processors. This happens because the parallel algorithm does not follow the same search strategy than the sequential one; this causes that the number of iterations and the number of function evaluations is different for both algorithms.

The algorithm PGSS2 gives the best results overall.

\section{Conclusions}

Three parallel Direct search methods have been implemented and described. Some new strategies have been applied:

1. In the GSS1 method, the new strategy of rotating the serch directions complements the strategy described in [6] of doubling the time step. This increases the probabilities of a descent direction being selected in two consecutive iterations, decreasing the average execution time.

2. In the GSS2 method, the addition of all the descent directions generates easily a new descent direction.

3. A Master-slave asynchronous algorithm was designed and implemented. It obtains quite good speed-ups, but the overall time execution is, with the present version, worse than the other two versions.

Finally, we would like to remark the great robustness shown by these methods in the IASVP problem, which is a really testing problem. For this problem, the convergence radius shown by these methods is far larger than the shown by Newton method, which, when converges, is much faster.

This shows that the Direct Search methods could be very useful for solution of difficult problems; if not for the full procedure, they could be used to obtain a good initial approximation for Newton's method, composing therefore a hybrid method. As shown, the long execution times of the Direct Search methods can be alleviated using parallel versions of these methods.

\section{Acknowledgement}

This work has been supported by Spanish MCYT and FEDER under Grant TIC2003-08238-C02-02 . 


\section{References}

1. Anderson E., Bai Z., Bishof C., Demmel J., Dongarra J.: LAPACK User Guide; Second edition. SIAM (1995)

2. Dennis J.E. and Torczon V.: Direct search methods on parallel machines; SIAM J. Optim., 1, (1991) 448-474

3. Flores F., García V.M., and Vidal A.M.: Numerical Experiments on the solution of the Inverse Additive Singular Value Problem; Computational Science - 5th International Conference, Atlanta, GA, USA (2005)

4. Hammarling S., Dongarra J., Du Croz J., Hanson R.J.: An extended set of fortran basic linear algebra subroutines; ACM Trans. Mat. Software (1988)

5. Hooke R. and Jeeves T.A.: Direct Search solution of numerical and statistical problems; Journal of the Association for Computing Machinery, (1961) 212-229

6. Hough P.D., Kolda T.G. and Torczon V.: Asyncronous parallel pattern for nonlinear optimization. SIAM J. Sci. Comput., 23, (2001) 134-156

7. C. T. Kelley: Iterative methods for linear and nonlinear equations; SIAM (1995).

8. Kolda T.G. and Torczon V.: On the Convergence of Asyncronous Parallel Pattern Search; Tech. Rep. SAND2001-8696, Sandia National Laboratories, Livermore, CA, (2002); SIAM J. Optim., submitted.

9. Kolda T. G., Lewis R. M. and Torczon V.: Optimization by Direct Search: New perspective on Some Clasical and Modern Methods; SIAM Review, Vol. 45, No. 3 (2003) 385-442

10. Kumar V., Gramar A., Gupta A. and Kerypis G.: Introduction to Parallel Computing: Desing and analysis of Algorithms; Redwood City, CA (1994)

11. Nelder J.A. and Mead R.: A simplex method for function minimization; The Computer Journal, 7 (1965) 308-313

12. Snir M., Otto S., Huss-Lederman S., Walker D. and Dongarra J.: MPI: The Complete Reference; MIT Press, (1996)

13. Torczon V.: Multi-Directional Search: A Direct Search Algorithm for Parallel Machines; Technical Report 90-7, Department of Computational and Applied Mathematics, Rice University, Houston, TX (1990), Author's 1989 Ph.D. dissertation. 